\title{
28 Research Square

\section{Mining Novel Specific Rumen Bacteria to Enhance the Bioactive Function of Mulberry Leaf in Promoting Host Antioxidant Activity and Immunomodulatory}

Bing Wang ( $\nabla$ wangb@cau.edu.cn )

China Agricultural University https://orcid.org/0000-0003-4410-0495

Hailing Luo

China Agricultural University

\section{Research}

Keywords: Antioxidant activity, Bioactivity, Morus alba, Microbiome, Rumen

Posted Date: June 29th, 2020

DOI: https://doi.org/10.21203/rs.3.rs-36704/v1

License: (c) (1) This work is licensed under a Creative Commons Attribution 4.0 International License. Read Full License 


\section{Abstract}

Background: Rumen is a natural fermentation system and the microorganisms inside can effectively utilize plant bioresource and interact with host metabolism. Here, analysis of rumen microbiome, together with animal performance and serum metabolism in a lamb model were performed to identify the potential use of mulberry leaf silage (MS) to replace alfalfa silage (AS) as a new functional feed resource and to mining the novel specific mulberry leaf associated rumen bacteria interact with host metabolism.

Results: The lambs fed with MS diet showed improved antioxidant capacity and immune function compared to those fed AS diet. The MS diet significantly altered rumen microbiota a- and $\beta$-diversity and taxonomic composition. Microbial analysis revealed that Bifidobacterium, Lactobacillus and Schwartzia were enhanced, and Ruminococcaceae UCG-010 and Lachnospiraceae_XPB1014_group were downregulated in the rumen of MS group. A strong association was also found between these rumen microbial taxa and host antioxidant capacity and immunomodulatory.

Conclusion: These findings indicated that mulberry leaf silage can be a high-quality feed source or bioactive pharmaceutical that is responsible for ruminants health benefits by modifying the rumen microbial community with potentially enhanced probiotics and inhibited biohydrogenation and methane emission.

\section{Background}

Mulberry (Morus alba) leaf, belonging to Moraceous plant, is commonly used as a Chinese traditional medicine for human beings and the sole food source for silkworms. It has a long history of cultivation throughout the world [1,2]. The mulberry leaf is a remarkable biomass production in China as its field area and production has been estimated to be 700,000 ha and approximately $1.82 \times 10^{7}$ t, respectively [3].

Mulberry leaf also has highly palatable and digestible (70-90\%) macronutrients to herbivorous animals, especially for its relative high protein content (15-28\%) and good essential amino acid profiles, which are similar to those of alfalfa hay $[4,5]$. Meanwhile, mulberry leaf has antioxidant, antibacterial, and immuneenhancing properties due to its abundant bioactive phytochemicals, such as polysaccharides, flavonoids, phenolic acid, and alkaloids, and thus can be used as an alternative "green" additive in properly replacing probiotics in improving immune function and preventing disease occurring [6-8]. Thus, mulberry leaf can be used as a functional feed source or feed supplement in the diets of ruminants and monogastric animal not only because of their abundant resources and exceptional nutritional value but with important biological activity $[8,9]$.

However, till now, mulberry leaves have not been fully utilized as animal feed source due to its high moisture content, but ensiling is an efficient method to solve this problem [10]. It has been confirmed that the fermented well silage has more palatable features for animal preference [11, 12]. Furthermore, the mulberry leaves after ensiling could hold robust bioactive activity such as antioxidant capacity and keep the total flavonoid content and even improve their bioactive activity by additives [13]. Several recent 
studies found that high-quality mulberry leaf silage can be achieved by additives [1, 10,13], but did not refer to the utilization of mulberry leaf silage. Rumen is a main position for the degradation of dietary nutrients in ruminants. The rumen microbiome is emerging as a cross bridge interacted with dietary utilization, host metabolism and phenotype changes, as shown by the association between rumen microbiome and the host metabolism [14-16].

Our questions focused on specific microbial species establishment in the rumen ecosystem induced by mulberry leaf silage, which might interact with the host metabolism in promoting antioxidant activity and immunomodulatory. Here, this study was to assess the mulberry leaf silage that could be a high-quality feed source with important biological activity and bio-feed resources for ruminants, and then to mining the associated rumen microbiome accounting for its bioactive function.

\section{Results}

\section{Growth and slaughter performance}

As shown in Table 1, DM intake, average daily gain (ADG), body weight (BW), and the ratio between DM intake and ADG were similar between the two diets. The slaughter BW, carcass weight, and dressing percentage were similar between the two groups. The weight of head and spleen in alfalfa silage diet (AS) group lambs were greater than those in mulberry leaf silage diet $(\mathrm{MS})$ group $(P<0.05)$. The weight of kidney in AS group lambs had a tendency to be lower than that in MS group $(P=0.053)$.

\section{Meat quality characteristics}

Meat quality characteristics were similar between the two diets, as shown in Table 1. Compared with the AS diet group, the lightness $\left(L^{*}\right)$ value at $45 \mathrm{~min}$ had a tendency to be lower in the MS diet group compare to AS group $(P=0.10)$.

\section{Nutrients metabolism, antioxidant activity, and immune response}

Overall, serum lipid metabolite levels including the concentrations of cholesterol, triacylglycerol, and the high density lipoprotein cholesterol (HDL) and low density lipoprotein cholesterol were un-affected by the MS diet (Table 2). The serum blood urea nitrogen (BUN) concentration was greater in the MS group than in the AS group $(P<0.01)$ but not for the serum albumin and globulin. As shown in Table 2 , the activity of serum catalase (CAT), glutathione peroxidase (GSH-PX), superoxide dismutase (SOD), and total antioxidant capacity (T-AOC) were significantly greater in the MS group than in the AS group $(P<0.05)$. The content of malondialdehyde (MDA)was significantly lower in the MS group than in the AS group ( $P<$ 0.05). Compared to the AS group, the serum interferon- $\gamma$ (IFN- $\gamma$ ) concentration was significantly increased in the MS group $(P<0.05)$. No significant difference was found for the tumor necrosis factor-a $($ TNF- $a)$ concentration between the two groups.

\section{Fermentation characteristics}


As shown in Table 3, rumen $\mathrm{pH}$ value and the concentrations of ammonia- $\mathrm{N}$ and total volatile fatty acids were similar between the AS and MS groups. The molar proportion of the isobutyrate had a tendency to be lower in the MS group than in the AS group $(P=0.09)$.

\section{Change in ruminal bacterial communities}

The Good's coverage of all samples was above 0.99. The Chao, Sobs, Shannon, and Ace indexes of bacterial richness and diversity were different between AS and MS (Table S2). The NMDS plots (Fig. 1A) showed that the clouds derived from the AS and MS data were clearly separated from each other with a significant stress value (0.026). There were 1,046 OTUs that were identified in both of the AS and MS groups, while 973 and 478 specific OTUs were observed in AS and MS groups, respectively (Fig. 1B). The anosim (analysis of similarities) based on bray-curtis distances showed significant different between the two groups $(P=0.007)$.

Five bacterial phyla were identified in the rumen samples that had relatively higher abundances $(>1 \%)$, including Bacteroidetes, Firmicutes, Proteobacteria, Actinobacteria, and Kiritimatiellaeota, among them, Bacteroidetes was significantly lower in MS group compare with AS group (Fig. 2A). There were 106 bacterial taxa identified at the genus level, and top 10 genera were present with relatively high abundances (Fig. 2B), including Prevotella_1, Selenomonas_1, Rikenellaceae_RC9_gut_group, Prevotella_7, Succiniclasticum, Bifidobacterium, Prevotellaceae_UCG-001, Succinivibrionaceae_UCG-001, Ruminococcus_2, and Veillonellaceae_UCG-001. We identified 23 genera as different bacteria based on the Wilcoxon rank-sum test (Fig. 3A), in which, 12 genera were more abundant in the MS samples, including Advenella, Tannerella, Succinivibrionaceae_UCG-001, Schwartzia, Bifidobacterium, Succinivibrio, Mitsuokella, Shuttleworthia, Olsenella, Howardella, Syntrophococcus, and Ruminococcus_gauvreauii_group. and 11 genera were with lower abundance in MS group, such as Prevotella_1, Erysipelotrichaceae_UCG-004, Ruminococcaceae_UCG-010, Fibrobacter, Ruminiclostridium_6, Oscillospira, Prevotellaceae_UCG-003, rumen_bacterium_YS3, Ruminococcaceae_UCG-001, Lachnospiraceae_XPB1014_group, and Lachnospiraceae_NK4A136_group. As shown in the non-strict version of LEfSe analysis (Fig. 3B, C), fourteen clades were more abundant in the MS samples, in which six genera were shown out as Howardella, Pelagibacterium, Protochlamydia, Shuttleworthia, Advenella, Planctomicrobium. Two clades were more abundant in the AS samples, in which the genus Lachnospiraceae_NK4A136_group was enriched.

It was found that the ruminal bacteria communities were related to the blood antioxidant activity ad immune function indices (Fig. 4). In detail, Lachnospiraceae_XPB1014_group and Ruminococcaceae_UCG-010 were both positively correlated with MDA $(r=0.73, P<0.01 ; r=0.70, P<$ $0.05)$, but negatively correlated with CAT $(r=-0.63, P<0.05 ; r=-0.66, P<0.05)$, GSH-PX $(r=-0.76, P<$ $0.01 ; \mathrm{r}=-0.64, P<0.05)$, SOD $(\mathrm{r}=-0.73, P<0.01 ; \mathrm{r}=-0.71, P<0.05)$, and T-AOC $(\mathrm{r}=-0.77, P<0.01 ; \mathrm{r}=$ $-0.70, P<0.05)$. Fibrobacter was positively correlated with MDA $(r=0.60, P<0.05)$, but negatively correlated with SOD $(r=-0.61, P<0.05)$ and T-AOC $(r=-0.60, P<0.05)$. The rumen_bacterium_YS3 was positively correlated with MDA $(r=0.68, P<0.05)$, but negatively correlated with GSH-PX $(r=-0.71, P<$ 
$0.01)$, SOD $(r=-0.66, P<0.05)$, and T-AOC $(r=-0.69, P<0.05)$. The Schwartzia was positively correlated with GSH-PX $(r=0.61, P<0.05)$, CAT $(r=0.61, P<0.05)$, and T-AOC $(r=0.58, P<0.05)$.

Ruminococcus_gauvreauii_group was positively correlated with SOD $(r=0.59, P<0.05)$.

Prevotellaceae_UCG-003 was positively correlated with MDA $(r=0.62, P<0.05)$, but negatively correlated with SOD $(r=-0.63, P<0.05)$, T-AOC $(r=-0.62, P<0.05)$, and IFN- $(r=-0.64, P<0.05)$. Bifidobacterium $(r$ $=0.76, P<0.01)$, Howardella $(r=0.72, P<0.01)$, and Olsenella $(r=0.62, P<0.05)$ were positively and Prevotella_ $1(\mathrm{r}=-0.75, P<0.01)$, was negatively correlated with IFN- $\mathrm{y}$.

\section{Discussion}

The biomass yield of fresh mulberry leaves is approximately 25 to 30 tons/ha/year, and the mulberry leaf is rich in protein (15-35\%) [3]. Therefore, mulberry leaves might be used as an excellent protein or feed supplement in animals $[3,17]$. In the current study, we found that the animal growth performance was maintained when mulberry leaf silage replaced alfalfa silage, which might be due to their similar protein level and digestible nutrients $[18,19]$. Furthermore, we also found that the mulberry leaf silage displays stronger antioxidant activities and immunomodulatory, which has not been reported in sheep. Oxidative stress, induced by reactive oxygen species and free radicals, is usually regarded as one of the causes of cell damage and even disease during nowadays high-intensive farming conditions, which inhibits ruminant growth and induces production loss [20,21]. Free radicals can be eliminated by antioxidant enzymes such as SOD, GSH-PX, CAT, etc. [22], thus the activities of antioxidant enzymes are a reflection of the antioxidative function of animal. The supplementation of exogenous antioxidants or utilizing plant source rich in antioxidants could be effective to prevent oxidative stress and improve animal health [21, 23]. It was reported mulberry leaves could be a source of natural antioxidants to combat oxidative stress in livestock production due to its scavenging of free radicals in animal feed [7]. Flavonoids and phenolic acids, presenting in mulberry leaf, were the large number of phytochemicals [24]. It was also found that the mulberry leaf is rich polyphenols, such as neochlorogenic acid, chlorogenic acid, rutin, quercetin, astragalin, and kaempferol [25], and the mulberry bioactive compounds showed the antioxidative capacity and anti-inflammatory effects $[13,26]$. The anti-oxidant effect of mulberry leaf flavonoids was associated with increased SOD, CAT and GSH-PX expression, as well as reduced MDA levels [27, 28]. It has been also reported that mulberry leaves could benefit animals through the enhancement of serum TAOC and the activities of CAT and SOD [7]. In the present study, the activity of serum CAT, GPX-PX, SOD, and T-AOC were increased and the serum MDA was reduced after lambs fed with mulberry leaf silage, confirming the improved antioxidant status in the Tan-lambs due to the preserved bioactive components in mulberry leaf silage [13].

The effective of mulberry leaf or its bioactive compounds in improving antioxidant capacity and immune function has been found in many previous studies what we talked above and was confirmed in the current study. To date, there have been few reports on the relationship between the effect roles of mulberry leaf silage on ruminal microbial community and the potential microbial targeted terminal function in antioxidant activity and immunomodulatory. In a previous study, the ruminal microbiota composition of finishing steers was not changed by partial replacement of corn grain and cotton seed 
meal with ensile mulberry leaves [29]. However, Tan et al. [30] reported that the inclusive of mulberry leaf in diet can not only stimulate the animal growth, but manipulate the ruminal microorganisms in cattle. In the current study, we found several genera bacteria were enhance or decreased by the feeding of mulberry leaf silage, of these changed bacteria, several of them also showed significantly correlated with host serum metabolism. The Prevotella accounts to the abundant genera in sheep rumen in the present study, which was also shown in the Hu-sheep [31]. It was also reported that the Prevotella genus has the ability in proteolytic [32]. It was found that ruminal Prevotella species may affect the amino acids metabolism in dairy cow [16]. We also found the improved serum BUN in MS group. Thus, the nitrogen utilization efficiency might be reduced by decreasing the abundance of Prevotella_ 1 in Tan-lambs fed with mulberry leaf silage.

In the current study, rumen Ruminococcac-eae_UCG_010 and Lachnospiraceae_XPB1014_group were down-regulated by feeding lambs mulberry leaf silage, which were also negatively correlated with serum antioxidant parameters. Ruminococcaceae UCG-010 is associated with ruminal biohydrogenation [33]. The abundances of Lachnospiraceae_XPB1014_group were negatively correlated with body fat weight in finishing pigs, indicating its function in lipid metabolism [34]. Thus, the reduced abundance of Ruminococcac-eae_UCG_010 and Lachnospiraceae_XPB1014_group in MS group indicated the inhibited lipid biohydrogenation in rumen. Thus, the mulberry leaf silage may have the ability to prevent the ruminal biohydrogenation by decreasing Ruminococcaceae UCG-010 and Lachnospiraceae_XPB1014_group.

It was also found that the Schwartzia was increased in the MS group compared to the AS group. The mulberry leaf flavonoid showed the ability in methane inhibiting in sheep [35]. Schwartzia was found to be negatively correlated with methane emissions [36]. Thus, the detected increased abundance of Schwartzia in the MS group from the current study and its positively correlated with antioxidant function might be due to the roles of Schwartzia in competing with methanogenic bacteria. Thus, these results may suggest the inhibited methane emission when lambs fed with mulberry leaf silage.

The immunoreaction is closely related to health of animals [37]. IFN- $y$ is involved in the initiation and regulation of the immune response [38]. It was found that water extracts of mulberry leaf mitigate inflammation through the interactions among insulin signalling pathway and TNF-a [39]. However, the current study did not find the changes of serum glucose and TNF-a, but with increased serum IFN- $y$ concentration shown in the current study. Mulberry leaves silage probably contains polyphenols and polysaccharides that could exert similar effects, as polyphenols and polysaccharides can inhibit inflammatory processes by improving serum IFN- $y$ concentration or affecting microbiota $[40,41]$. In addition, cumulative evidences indicate that mulberry leaf polysaccharides have immunomodulating activity, which has been assessed on many different kind cells and macrophage-dependent immune system responses [42]. Here, we show, to our knowledge for the first time, the ruminal Bifidobacterium and Lactobacillus genera were increased by feeding mulberry leaf silage in sheep which were also positively correlated with the serum IFN- $y$ concentration in the current study. It was found the bacterial supernatants of the Bifidobacterium and Lactobacillus genera, are able to affect ghrelin receptor (growth 
hormone secretagogue receptor-1a) signaling, which plays a crucial role in maintaining energy balance and metabolism, and modulating food intake, motivation, reward, and mood in human being [43]. Thus, in total, we estimated that the improved level of Bifidobacterium and Lactobacillus genera in rumen of MS group might contribute to the improved healthy level and status of lambs fed with mulberry leaf silage.

The increased Bifidobacterium in rumen of lamb were associated with the improved contents of meat nutrients comprising alpha-linolenic acid, conjugated linoleic acid and eicosapentaenoic acid [44]. Lactobacilli and Bifidobacterium are considered by the European Food Safety Authority, indicating they can be considered possible prebiotics for improvement of gut health [45]. Bifidobacteria was abundant in the digestive tract of humans, which has assumed health-promoting activities [46]. Vlková et al. [47] also found that the supplementation of Bifidobacterium strains promoted rumen health for calves in the milkfeeding period. On the other hand, Bifidobacteria can utilize a variety of isoflavones, mono- and oligosaccharide, and polysaccharides [45]. Mulberry leaf contains abundant polysaccharides and other plant secondary metabolites [41,48], thus the increased abundance of rumen Bifidobacteria in the MS group might due to the feeding of mulberry leaf silage. Our findings for the increased abundance of rumen Bifidobacteria in MS group compared to AS group and its highly positively correlation with serum IFN- $\gamma$ seem to indicate the beneficial effects of genus Bifidobacterium on Tan-sheep.

Additionally, except for the described potentially differential functional bacterial, we observed that Fibrobacter, Lachnospiraceae_AC2044_group, Prevotellaceae UCG-003, and rumen_bacterium_YS3 were negatively correlated with the enhancement of serum antioxidant characteristics and immune function. In addition, we also found the increased abundance of Howardella, Pelagibacterium, Protochlamydia, Shuttleworthia, Advenella, and Planctomicrobium were achieved in the MS group. However, we did not find the related function of these bacteria. More attention should be paid in detecting their function in further studies by screening and culture methods, as most of their potential function of them in restricting rumen health and host health is still unknown. In total, our research provides a preliminary view of the efficient utilization of mulberry leaf silage in lambs, and we detect several potential mulberry leaf silageassociated rumen bacteria that may interact with the host metabolism and account for the enhanced antioxidant capacity and immunomodulatory.

\section{Materials And Methods}

\section{Silage preparation}

Alfalfa and mulberry were cultivated and harvested on Wuzhong, Ningxia Hui Autonomous Region $\left(37.99^{\circ} \mathrm{N}, 106.20^{\circ} \mathrm{E}\right)$. The fresh alfalfa and mulberry leaf were collected and chopped to $2-3 \mathrm{~cm}$ using a forage cutter (Lingong Machinery, Shandong, China). Then, both of the alfalfa and mulberry leaf were mixed with $1 \%$ glucose and ensiled with $1 \times 10^{6}$ colony forming units $(\mathrm{cfu}) / \mathrm{g}$ of fresh material Lactobacillus plantarum (GenBank accession number: WCFS1) [49]. Both of these two kind silages were fermented well after $60 \mathrm{~d}$ ensiling based on the sensory evaluation and $\mathrm{pH}$ value measured by a $\mathrm{pH}$ meter 
(PHS-3C, Shanghai Leijun Experimental Instrument Co., Ltd., Shanghai, China) after the bale silage opening. Then, the ingredients were sampled and analyzed. The dry matter (DM) content of the alfalfa silage and mulberry leaf silage were 32.9 and $28.7 \%$, respectively. The concentrations of crude protein $(C P)$, neutral detergent fiber (NDF), acid detergent fiber (ADF), ash, and ether extract (EE) in alfalfa silage were $15.9,56.6,37.4,14.6$ and $1.68 \%$ of the DM basis, respectively. The concentrations of CP, NDF, ADF, ash, and EE in mulberry leaf silage were $16.2,63.4,39.2,14.3$ and $2.87 \%$ of the DM basis, respectively.

\section{Experimental design and sampling}

Forty Tan lambs (Ovis aries) with average age of $75 \mathrm{~d}( \pm 3)$, BW of $15.3 \mathrm{~kg}( \pm 1.92 \mathrm{SD})$ were selected from were selected from 5,000 sheep fed in Tianyuan Liangzhong Sheep Farm (Ningxia Hui Autonomous Region, China). The sheep were blocked into 20 groups based on the BW and were randomly allocated within the blocks to 1 of 2 treatments (on a DM basis): 1) alfalfa silage based diet (AS; a diet containing $20 \%$ alfalfa silage, $n=20$ ), 2) mulberry leaf silage based diet (MS; a diet containing $20 \%$ mulberry leaf silage, $n=20$ ), the other ingredients were identical (Table S1). The animals in each diet group were randomly placed in 4 pens with 5 lambs in each pen. The two groups lambs were both fed with a basal total mixed ration diet. The AS and MS diets contain (DM basis), 14.6 and $14.7 \%$ of $C P, 31.1$ and $32.4 \%$ of NDF, $17.8 \%$ and $18.2 \%$ of $A D F, 4.70 \%$ and $4.94 \%$ of $E E, 6.20$ and $6.15 \%$ of ash, respectively. The feed intake was recorded daily and was measured based on the difference between the amount of feed deliveries and the remaining. The experiment lasted for 80 days, including a 20 days' adaptation and 60 days' formal feeding. All the lambs had ad libitum access to feed and water.

The BW of each lambs were recorded every 20 days. On the day 80 (the end of the experiment), six lambs in each group were then selected for blood sampling and slaughtering based on the average BW. Blood was collected from the jugular vein of the sheep to separate serum (centrifuged for 10 min at 3,000 $\times \mathrm{g}$ ). After slaughtering, the weight of carcass, head, skin, limbs, internal organs (heart, liver, spleen, lung, kidney) were measured and recorded. All the collected samples were then stored at liquid nitrogen for subsequent analysis.

The detail information of the meat quality detection was according to a previous study of Liang et al. [50]. In brief, body fat (assessed as GR) value was assessed by measuring the thickness at the 12th/13th rib intersection $110 \mathrm{~mm}$ away from the midline using a vernier caliper. The $\mathrm{pH}$ value and meat colour (redness $\left(a^{*}\right)$, yellowness $\left(b^{\star}\right)$, and lightness $\left(L^{*}\right)$, psychometric chroma $\left(c^{*}=\left(a^{2}+b^{2}\right)^{0.5}\right)$, and Hue angle $\left(H^{\star}=\arctan \left(b^{\star} / a^{\star}\right) \times(180 / \pi)\right)$ at $45 \mathrm{~min}$ and $24 \mathrm{~h}$ were measured. Each sample was determined three times at different positions on the meat surface and the average value was obtained. Serum metabolite concentrations, including total protein, albumin, BUN, triacylglycerol, total cholesterol, high density lipoprotein cholesterol, low density lipoprotein cholesterol, immunoglobin $G$, immunoglobin $A$, and immunoglobin $M$ were measured using an automatic analyzer (Kehua ZY KHB-1280, Shanghai, China) with a commercial kits (Shanghai Kehua Biological Technology Co. Ltd., Shanghai, China) [51]. The activities of antioxidant enzymes such as SOD, CAT, GSH-PX, T-AOC and MDA, and the concentrations of 
IFN- $y$ and TNF-a were determined by commercial kits (Jiancheng Biological Technology Co. Ltd., Nanjing, China) following the manufacturer's instructions.

Rumen fluid was collected from the ventral part of the rumen by straining the ruminal content through four layers of cheesecloth. Rumen fluid $\mathrm{pH}$ was measured immediately. Rumen fluid samples were then stored at liquid nitrogen for subsequent analysis. The concentrations of volatile fatty acids were measured by gas chromatography (Trace 1300; Thermo Fisher Scientific Co., Ltd., Shanghai, China). The ammonia nitrogen was determined by the method described in Broderick and Kang [52].

\section{Profiling of rumen bacterial community diversity}

Microbial DNA from rumen fluid was extracted by a HiPure Stool DNA Kit (Magen, Guangzhou, China) according to manufacturer's protocols. The 16S rDNA V3-V4 region of the ribosomal RNA gene were amplified by PCR using primers 341F: CCTACGGGNGGCWGCAG; 806R: GGACTACHVGGGTATCTAAT following the procedure described by Sun et al. [53]. The amplicons were purified by the AxyPrep DNA Gel Extraction Kit (Axygen Biosciences, Union City, CA, U.S.). Then it was quantified by ABI StepOnePlus RealTime PCR System (Life Technologies, Foster City, USA).

Purified amplicons were pooled in equimolar and paired-end sequenced (PE250) on an Illumina platform (Illumina Novaseq 6000 sequencing) according to the standard protocols. Raw reads were further filtered using FASTP to get high quality clean reads [54]. The noisy sequences of raw tags were filtered by QIIME (version 1.9.1) [55]. Then, chimeric tags were removed using UCHIME algorithm. The finally obtained effective tags were clustered into operational taxonomic units (OTUs) of $\geq 97 \%$ similarity. Based on the SILVA database (version 132), the representative sequences were assigned to organisms by a naive Bayesian model using RDP classifier (Version 2.2). The abundance statistics of each taxonomy were constructed using a Perl script and visualized by SVG. The alpha index including Chao, Simpson, Sobs, Shannon, and Ace were calculated in QIIME. Unweighted non-metric multi-dimensional scaling (NMDS) was generated in R project Vegan package (version 2.5.3). Statistical analysis of Wilcoxon rank test and Anosim test was calculated in $\mathrm{R}$ project Vegan package (version 2.5.3). The bacterial community comparison between the AS and MS groups was calculated by Wilcoxon rank test in R project Vegan package (version 2.5.3). Pearson correlation coefficient between environmental factors and species and between blood parameters and species was calculated in R project psych package (version 1.8.4).

Network of these correlation coefficients were generated using igraph package (version 1.1.2) in R project.

\section{Statistical analyses}

The animal performance, blood parameters, and rumen fermentation characteristics data were analyzed for a completely random design using the PROC MIXED procedure of SAS (version 9.4, SAS Institute Inc., Cary, NC). The means of each treatments are presented as least squares means and statistical significance was defined at $P<0.05$, and the trends were declared at $0.05 \leq P \leq 0.10$.

\section{Conclusions}


We here identify a potent effect of mulberry leaf silage-associated ruminal microbiota interact with host metabolism and physiology. The enriched ruminal Bifidobacterium, Lactobacillus, and Schwartzia, and the down-regulated ruminal Ruminococcaceae UCG-010 and Lachnospiraceae_XPB1014_group in the MS group lambs might contribute to the bioconversion and bioactive function of mulberry leaf silage in rumen. Furthermore, these specific mulberry leaf silage-associated bacteria were associated with the enhanced animal antioxidant capacity or immunomodulatory. Altogether, evidence from the results of the current study suggest that the naturally mulberry leaf silage could be an alternative functional supplement in maintaining animal health by rescheduling the rumen bacterial community.

\section{List of abbreviations}

$A D G$, average daily gain; ADF, acid detergent fiber; AS, alfalfa silage diet; BUN, blood urea nitrogen; CAT, catalase; $\mathrm{CP}$, crude protein; DM, dry matter; EE, ether extract; GSH-PX, glutathione peroxidase; MDA, malondialdehyde; MS, mulberry leaf silage diet; NDF, neutral detergent fiber; SOD, superoxide dismutase; T-AOC, total antioxidant capacity.

\section{Supplementary information}

Supplementary information accompanies this paper at

Additional file 1: Table S1. Ingredients and nutrient composition of the total mixed ration containing alfalfa silage (AS) and mulberry leaf silage (MS) as the main forage. Table S2. Alpha diversity indices of rumen bacteria in lambs fed alfalfa silage (AS) and mulberry leaf silage (MS) based diets.

\section{Abbreviations}

$A D G$, average daily gain; ADF, acid detergent fiber; AS, alfalfa silage diet; BUN, blood urea nitrogen; CAT, catalase; $\mathrm{CP}$, crude protein; $\mathrm{DM}$, dry matter; $\mathrm{EE}$, ether extract; GSH-PX, glutathione peroxidase; MDA, malondialdehyde; MS, mulberry leaf silage diet; NDF, neutral detergent fiber; SOD, superoxide dismutase; T-AOC, total antioxidant capacity.

\section{Declarations}

\section{Acknowledgements}

We greatly acknowledge the personnel of Tianyuan Liangzhong Sheep Farm (Wuzhong, China) for their animal feeding.

\section{Authors' contributions}

BW and HL conceived and designed the study; BW carried out the field and laboratory work and bioinformatic analyses. BW drafted the manuscript; $\mathrm{HL}$ coordinated the study and helped draft the 
manuscript. All authors gave final approval for publication and agree to be held accountable for the work performed therein.

\section{Funding}

This study was funded by the Demonstration Project of Exploitation and Utilization of High-Quality Green and Rough Feed Resources Development (16190051), the National Natural Science Foundation of China (31902181), and the College and Discipline Autonomous Projects of China Agricultural University (2020TC065).

\section{Availability of data and materials}

The data sets generated during and/or analysed during the current study are available from the corresponding author on reasonable request. The raw reads of $16 \mathrm{~S}$ rRNA sequencing data have been deposited into the NCBI Sequence Read Archive (SRA) database (Accession Number: SRP265735).

\section{Ethical approval and consent to participate}

The animals used in this study were approved by the Animal Care Committee of China Agricultural University (Beijing, China; approval no. AW30129102-1-1; approval date: 26 October 2019). The detail experimental procedures were following the university's guidelines for animal research.

\section{Consent for publication}

Our study does not contain data from any individual person. Hence this section is not applicable.

\section{Competing interests}

The authors declare no competing financial interest.

\section{Author details}

State Key Laboratory of Animal Nutrition, College of Animal Science and Technology, China Agricultural University, Beijing 100193, P. R. China.

\section{References}

1. Wang Y, Chen X, Wang C, He L, Zhou W, Yang F, Zhang Q. The bacterial community and fermentation quality of mulberry (Morus alba) leaf silage with or without Lactobacillus casei and sucrose. Bioresour Technol. 2019;293:122059.

2. Wen P, Hu TG, Linhardt RJ, Liao ST, Wu H, Zou YX. Mulberry: A review of bioactive compounds and advanced processing technology. Trends Food Sci Tech. 2019;83:138-58.

3. Kandylis K, Hadjigeorgiou I, Harizanis P. The nutritive value of mulberry leaves (Morus alba) as a feed supplement for sheep. Trop Anim Health Prod. 2009;41:17-24. 
4. Doran MP, Laca EA, Sainz RD. Total tract and rumen digestibility of mulberry foliage (Morus alba), alfalfa hay and oat hay in sheep. Anim Feed Sci Tech. 2007;138:239-53.

5. Jetana T, Vongpipatana C, Usawang S, Thongruay S. The use of tropical protein-rich leaves as supplements to Thai swamp buffalo receiving a basal diet of rice straw and treated leucaena (Leucaena leucocephala). Trop Anim Health Prod. 2011;43:57-67.

6. Butt MS, Nazir A, Sultan MT, Schroen K. Morus alba L. nature's functional tonic. Trends Food Sci Tech. 2008;19:505-12.

7. Lin WC, Lee MT, Chang SC, Chang YL, Shih CH, Yu B, Lee TT. Effects of mulberry leaves on production performance and the potential modulation of antioxidative status in laying hens. Poult Sci. 2017;96:1191-203.

8. Zhao X, Li L, Luo Q, Ye M, Luo G, Kuang Z. Effects of mulberry (Morus alba L.) leaf polysaccharides on growth performance, diarrhea, blood parameters, and gut microbiota of early-weanling pigs. Livest Sci. 2015;177:88-94.

9. Cai M, Mu L, Wang ZL, Liu JY, Liu TL, Wanapat M, Huang BZ. Assessment of mulberry leaf as a potential feed supplement for animal feeding in P.R. China. Asian-Australas J Anim Sci. 2019;32:1145-52.

10. He L, Chen N, Lv H, Wang C, Zhou W, Chen X, Zhang Q. Gallic acid influencing fermentation quality, nitrogen distribution and bacterial community of high-moisture mulberry leaves and stylo silage. Bioresour Technol. 2020;295:122255.

11. Kung L Jr, Shaver RD, Grant RJ, Schmidt RJ. Silage review: Interpretation of chemical, microbial, and organoleptic components of silages. J Dairy Sci. 2018;101:4020-33.

12. Muller CE, Uden P. Preference of horses for grass conserved as hay, haylage or silage. Anim Feed Sci Tech. 2007;132:66-78.

13. He L, Zhou W, Wang C, Yang F, Chen X, Zhang Q. Effect of cellulase and Lactobacillus casei on ensiling characteristics, chemical composition, antioxidant activity, and digestibility of mulberry leaf silage. J Dairy Sci. 2019;102:9919-31.

14. Li F, Li C, Chen Y, Liu J, Zhang C, Irving B, Fitzsimmons C, Plastow G, Guan LL. Host genetics influence the rumen microbiota and heritable rumen microbial features associate with feed efficiency in cattle. Microbiome. 2019;7:92.

15. Lin L, Xie F, Sun D, Liu J, Zhu W, Mao S. Ruminal microbiome-host crosstalk stimulates the development of the ruminal epithelium in a lamb model. Microbiome. 2019;7:83.

16. Xue MY, Sun HZ, Wu XH, Liu JX, Guan LL. Multi-omics reveals that the rumen microbiome and its metabolome together with the host metabolome contribute to individualized dairy cow performance. Microbiome. 2020;8:64.

17. Liu JX, Yao J, Yan B, Yu JQ, Shi ZQ. Effects of mulberry leaves to replace rapeseed meal on performance of sheep feeding on ammoniated rice straw diet. Small Rumin Res. 2001;39:131-6.

18. Cornara L, Xiao J, Burlando B. Therapeutic Potential of Temperate Forage Legumes: A Review. Crit Rev Food Sci Nutr. 2016;56(Suppl 1):149-61. 
19. Ji T, Li J, Su SL, Zhu ZH, Guo S, Qian DW, Duan JA. Identification and Determination of the Polyhydroxylated Alkaloids Compounds with alpha-Glucosidase Inhibitor Activity in Mulberry Leaves of Different Origins. Molecules 2016, 21.

20. Memon MA, Wang Y, Xu T, Ma N, Zhang H, Roy AC, Aabdin ZU, Shen X. Lipopolysaccharide induces oxidative stress by triggering MAPK and Nrf2 signalling pathways in mammary glands of dairy cows fed a high-concentrate diet. Microb Pathog. 2019;128:268-75.

21. Nisar NA, Sultana M, Waiz HA, Para PA, Dar SA. Oxidative Stress - Threat to Animal Health and Production. Int J Livest Res. 2013;3:76-83.

22. Kurata M, Suzuki M, Agar NS. Antioxidant systems and erythrocyte life-span in mammals. Comp Biochem Physiol B. 1993;106:477-87.

23. Pandey V, Singh A, Singh A, Krishna A, Pandey U, Tripathi YB. Role of oxidative stress and low-grade inflammation in letrozole-induced polycystic ovary syndrome in the rat. Reprod Biol. 2016;16:70-7.

24. Sanchezsalcedo EM, Mena P, Garciaviguera C, Hernandez F, Martinez JJ. (Poly)phenolic compounds and antioxidant activity of white (Morus alba) and black (Morus nigra) mulberry leaves: Their potential for new products rich in phytochemicals. J Funct Foods. 2015;18:1039-46.

25. Lee WJ, Choi SW. Quantitative Changes of Polyphenolic Compounds in Mulberry (Morus alba L.) Leaves in Relation to Varieties, Harvest Period, and Heat Processing. Prev Nutr Food Sci. 2012;17:280-5.

26. Gao XH, Zhang SD, Wang LT, Yu L, Zhao XL, Ni HY, Wang YQ, Wang JD, Shan CH, Fu YJ. AntiInflammatory Effects of Neochlorogenic Acid Extract from Mulberry Leaf (Morus alba L.) Against LPS-Stimulated Inflammatory Response through Mediating the AMPK/Nrf2 Signaling Pathway in A549 Cells. Molecules 2020, 25.

27. Wang Y, Li M, Yu X, He S, Wu X, Wang Y. Mulberry leaf flavonoids protect against glucotoxicityinduced INS-1 cell apoptosis. J Tradit Chin Med. 2019;39:153-9.

28. Yang S, Wang BL, Li Y. Advances in the pharmacological study of Morus alba L. Yao Xue Xue Bao. 2014;49:824-31.

29. Niu Y, Meng Q, Li S, Ren L, Zhou B, Schonewille T, Zhou Z. Effects of Diets Supplemented with Ensiled Mulberry Leaves and Sun-Dried Mulberry Fruit Pomace on the Ruminal Bacterial and Archaeal Community Composition of Finishing Steers. PLoS One. 2016;11:e0156836.

30. Tan ND, Wanapat M, Uriyapongson S, Cherdthong A, Pilajun R. Enhancing mulberry leaf meal with urea by pelleting to improve rumen fermentation in cattle. Asian-Australas J Anim Sci. 2012;25:45261.

31. Yang B, Le J, Wu P, Liu J, Guan LL, Wang J. Alfalfa Intervention Alters Rumen Microbial Community Development in Hu Lambs During Early Life. Front Microbiol. 2018;9:574.

32. Matsui H, Ogata K, Tajima K, Nakamura M, Nagamine T, Aminov RI, Benno Y. Phenotypic characterization of polysaccharidases produced by four Prevotella type strains. Curr Microbiol. 2000;41:45-9. 
33. Opdahl LJ, Gonda MG, St-Pierre B. Identification of Uncultured Bacterial Species from Firmicutes, Bacteroidetes and CANDIDATUS Saccharibacteria as Candidate Cellulose Utilizers from the Rumen of Beef Cows. Microorganisms 2018, 6.

34. Hu C, Li F, Duan Y, Yin Y, Kong X. Glutamic acid supplementation reduces body fat weight in finishing pigs when provided solely or in combination with arginine and it is associated with colonic propionate and butyrate concentrations. Food Funct. 2019;10:4693-704.

35. Ma T, Chen DD, Tu Y, Zhang NF, Si BW, Diao QY. Dietary supplementation with mulberry leaf flavonoids inhibits methanogenesis in sheep. Anim Sci J. 2017;88:72-8.

36. Cunha CS, Veloso CM, Marcondes MI, Mantovani HC, Tomich TR, Pereira LGR, Ferreira MFL, DillMcFarland KA, Suen G. Assessing the impact of rumen microbial communities on methane emissions and production traits in Holstein cows in a tropical climate. Syst Appl Microbiol. 2017;40:492-9.

37. Ingvartsen KL, Moyes K. Nutrition, immune function and health of dairy cattle. Animal. 2013;7(Suppl 1):112-22.

38. Kuang Y, Wang Y, Zhang Y, Song Y, Zhang X, Lin Y, Che L, Xu S, Wu D, Xue B. Effects of dietary combinations of organic acids and medium chain fatty acids as a replacement of zinc oxide on growth, digestibility and immunity of weaned pigs. Anim Feed Sci Tech. 2015;208:145-57.

39. Tian S, Wang M, Liu C, Zhao H, Zhao B. Mulberry leaf reduces inflammation and insulin resistance in type 2 diabetic mice by TLRs and insulin Signalling pathway. BMC Complement Altern Med. 2019;19:326.

40. Gessner DK, Bonarius M, Most E, Fiesel A, Eder K. Effects of polyphenol-rich plant products from grape or hop as feed supplements on the expression of inflammatory, antioxidative, cytoprotective and endoplasmic reticulum stress-related genes and the antioxidative status in the liver of piglets. J Anim Physiol Anim Nutr (Berl). 2017;101:e185-94.

41. Zhao X, Yang R, Bi Y, Bilal M, Kuang Z, Iqbal HMN, Luo Q. Effects of Dietary Supplementation with Mulberry (Morus alba L.) Leaf Polysaccharides on Immune Parameters of Weanling Pigs. Animals (Basel) 2019, 10.

42. He X, Fang J, Ruan Y, Wang X, Sun Y, Wu N, Zhao Z, Chang Y, Ning N, Guo H, Huang L. Structures, bioactivities and future prospective of polysaccharides from Morus alba (white mulberry): A review. Food Chem. 2018;245:899-910.

43. Torres-Fuentes C, Golubeva AV, Zhdanov AV, Wallace S, Arboleya S, Papkovsky DB, El Aidy S, Ross P, Roy BL, Stanton C, et al. Short-chain fatty acids and microbiota metabolites attenuate ghrelin receptor signaling. FASEB J. 2019;33:13546-59.

44. Lyons T, Boland T, Storey S, Doyle E. Linseed Oil Supplementation of Lambs' Diet in Early Life Leads to Persistent Changes in Rumen Microbiome Structure. Front Microbiol. 2017;8:1656.

45. Perez-Burillo S, Rajakaruna S, Pastoriza S, Paliy O, Angel Rufian-Henares J. Bioactivity of food melanoidins is mediated by gut microbiota. Food Chem. 2020;316:126309. 
46. Slovakova L, Duskova D, Marounek M. Fermentation of pectin and glucose, and activity of pectindegrading enzymes in the rabbit caecal bacterium Bifidobacterium pseudolongum. Lett Appl Microbiol. 2002;35:126-30.

47. Vlkova E, Trojanova I, Rada V. Distribution of bifidobacteria in the gastrointestinal tract of calves. Folia Microbiol (Praha). 2006;51:325-8.

48. Chan EW, Lye PY, Wong SK. Phytochemistry, pharmacology, and clinical trials of Morus alba. Chin J Nat Med. 2016;14:17-30.

49. Zhang Q, Li XJ, Zhao MM, Yu Z. Isolating and evaluating lactic acid bacteria strains for effectiveness of Leymus chinensis silage fermentation. Lett Appl Microbiol. 2014;59:391-7.

50. Liang Y, Bao Y, Gao X, Deng K, An S, Wang Z, Huang X, Liu D, Liu Z, Wang F, Fan Y. Effects of spirulina supplementation on lipid metabolism disorder, oxidative stress caused by high-energy dietary in $\mathrm{Hu}$ sheep. Meat Sci. 2020;164:108094.

51. Fan Y, Ren C, Meng F, Deng K, Zhang G, Wang F. Effects of algae supplementation in high-energy dietary on fatty acid composition and the expression of genes involved in lipid metabolism in $\mathrm{Hu}$ sheep managed under intensive finishing system. Meat Sci. 2019;157:107872.

52. Broderick G, Kang J. Automated simultaneous determination of ammonia and total amino acids in ruminal fluid and in vitro media1. J Dairy Sci. 1980;63:64-75.

53. Sun Z, Yu Z, Wang B. Perilla frutescens Leaf Alters the Rumen Microbial Community of Lactating Dairy Cows. Microorganisms 2019, 7.

54. Chen S, Zhou Y, Chen Y, Gu J. fastp: an ultra-fast all-in-one FASTQ preprocessor. Bioinformatics. 2018;34:i884-90.

55. Bokulich NA, Subramanian S, Faith JJ, Gevers D, Gordon JI, Knight R, Mills DA, Caporaso JG. Qualityfiltering vastly improves diversity estimates from Illumina amplicon sequencing. Nat Methods. 2013;10:57-9.

\section{Tables}

Table 1 Effect of alfalfa silage (AS) and mulberry leaf silage (MS) based diets on growth performance, organ index, and meat quality characteristics in lambs 


\begin{tabular}{|c|c|c|c|c|}
\hline \multirow[t]{2}{*}{ Items } & \multicolumn{4}{|c|}{ Treatments } \\
\hline & AS & MS & SEM & $P$-value \\
\hline \multicolumn{5}{|l|}{ Growth performance } \\
\hline DMI, g/d & 0.62 & 0.61 & 0.007 & 0.67 \\
\hline Od BW, & 15.2 & 15.3 & 0.44 & 0.90 \\
\hline $80 \mathrm{~d} \mathrm{BW}, \mathrm{kg}$ & 21.2 & 22.0 & 0.36 & 0.17 \\
\hline$A D G, g / d$ & 97.7 & 96.0 & 7.85 & 0.87 \\
\hline DMl/ADG, $g / g$ & 6.34 & 6.39 & 0.378 & 0.92 \\
\hline \multicolumn{5}{|l|}{ Organ index } \\
\hline Carcass weight, kg & 10.5 & 10.7 & 0.23 & 0.57 \\
\hline Dressing percentage, $\%$ & 49.5 & 48.5 & 0.44 & 0.19 \\
\hline Head weight, kg & 1.55 & 1.37 & 0.042 & 0.03 \\
\hline Hooves weight, kg & 0.48 & 0.48 & 0.018 & 1.00 \\
\hline Pelage weight, kg & 1.83 & 1.92 & 0.072 & 0.45 \\
\hline Heart, kg & 89.8 & 84.4 & 3.99 & 0.38 \\
\hline Liver, kg & 318.6 & 338.5 & 21.08 & 0.53 \\
\hline Spleen, kg & 32.5 & 29.2 & 0.72 & 0.02 \\
\hline Lung, kg & 199.4 & 197.8 & 6.30 & 0.87 \\
\hline Kidney, kg & 30.1 & 34.5 & 1.22 & 0.05 \\
\hline Tail lipid, kg & 720.0 & 835.6 & 54.09 & 0.19 \\
\hline Kidney lipid, kg & 38.4 & 44.5 & 4.85 & 0.41 \\
\hline \multicolumn{5}{|l|}{ Meat quality } \\
\hline $\mathrm{GR}, \mathrm{mm}$ & 12.9 & 13.4 & 0.56 & 0.55 \\
\hline $\mathrm{pH} 45 \mathrm{~min}$ & 6.83 & 6.70 & 0.075 & 0.27 \\
\hline$a * 45 \mathrm{~min}$ & 6.27 & 6.81 & 0.394 & 0.38 \\
\hline$b * 45 m i n$ & 6.68 & 6.84 & 0.748 & 0.89 \\
\hline$c^{\star} 45 \mathrm{~min}$ & 9.48 & 9.80 & 0.743 & 0.77 \\
\hline $\mathrm{H}^{\star} 45 \mathrm{~min}$ & 46.4 & 44.3 & 2.27 & 0.53 \\
\hline$L * 45 \min$ & 34.1 & 32.2 & 0.67 & 0.10 \\
\hline
\end{tabular}




\begin{tabular}{|lllll|}
$\mathrm{pH} 24 \mathrm{~h}$ & 5.66 & 5.55 & 0.074 & 0.32 \\
\hline $\mathrm{a} * 24 \mathrm{~h}$ & 8.60 & 9.18 & 0.520 & 0.46 \\
\hline $\mathrm{b} * 24 \mathrm{~h}$ & 10.7 & 11.1 & 0.68 & 0.71 \\
\hline $\mathrm{c} * 24 \mathrm{~h}$ & 13.8 & 14.4 & 0.80 & 0.59 \\
\hline $\mathrm{H}^{*} 24 \mathrm{~h}$ & 51.4 & 49.9 & 1.17 & 0.42 \\
\hline $\mathrm{L} * 24 \mathrm{~h}$ & 41.2 & 41.0 & 0.62 & 0.78 \\
\hline
\end{tabular}

$\mathrm{DMI}=$ dry matter intake; $\mathrm{BW}$ = body weight; $\mathrm{ADG}=$ average daily gain; $\mathrm{GR}=$ the depth of muscle and fat tissue from the surface of the carcass to the lateral surface of the 12th rib $110 \mathrm{~mm}$ from the midline; $\mathrm{a}^{*}=$ redness; $b^{*}=$ yellowness; $\mathrm{C}^{*}=$ Chroma; $\mathrm{H}^{*}=$ Hue angle; $\mathrm{L}^{*}=$ lightness; $\mathrm{SEM}=$ standard error of means.

Table 2 Effect of alfalfa silage (AS) and mulberry leaf silage (MS) based diets on serum biochemical, antioxidant, and immune characteristics in lambs 


\begin{tabular}{|lllll|}
\hline \multicolumn{5}{l}{ Treatments } \\
\hline Items & AS & MS & SEM & P-value \\
\hline Total protein, g/L & 63.0 & 64.2 & 1.19 & 0.49 \\
\hline Albumin, g/L & 28.9 & 28.7 & 0.42 & 0.78 \\
\hline Globulin, g/L & 34.1 & 35.5 & 0.83 & 0.28 \\
\hline BUN, mmol/L & 6.64 & 7.00 & 0.061 & 0.01 \\
\hline Glucose, mmol/L & 5.06 & 5.34 & 0.275 & 0.50 \\
\hline Total cholesterol, mmol/L & 1.47 & 1.57 & 0.143 & 0.63 \\
\hline Triglyceride, mmol/L & 0.60 & 0.67 & 0.059 & 0.42 \\
\hline HDL, mmol/L & 0.54 & 0.57 & 0.048 & 0.63 \\
\hline LDL, mmol/L & 0.81 & 0.86 & 0.084 & 0.64 \\
\hline IgA, g/L & 0.64 & 0.61 & 0.018 & 0.37 \\
\hline IgG, g/L & 17.2 & 17.6 & 0.17 & 0.19 \\
\hline IgM, g/L & 1.13 & 1.12 & 0.057 & 0.90 \\
\hline CAT, U/ml & 12.2 & 12.7 & 0.09 & 0.02 \\
\hline GSH-PX, U/ml & 952.4 & 979.4 & 6.79 & 0.04 \\
\hline MDA, nmol/ml & 4.96 & 4.54 & 0.098 & 0.03 \\
\hline SOD, U/ml & 94.1 & 97.4 & 0.82 & 0.03 \\
\hline T-AOC, U/ml & 9.99 & 10.57 & 0.141 & 0.03 \\
\hline TNF-a, pg/ml & 44.9 & 45.9 & 0.70 & 0.34 \\
\hline IFN-y, pg/ml & 154.5 & 157.7 & 0.67 & 0.02 \\
\hline
\end{tabular}

BUN = blood urea nitrogen; $\mathrm{HDL}$ = high-density lipoprotein cholesterol; $L D L=$ low-density lipoprotein cholesterol; IgA = immunoglobin A; IgG = immunoglobin $\mathrm{G}$; IgM = immunoglobin $\mathrm{M}$; CAT = catalase; $\mathrm{GSH}$ $\mathrm{PX}=$ glutathione peroxidase; $\mathrm{MDA}=$ malondialdehyde; $\mathrm{SOD}=$ superoxide dismutase; $\mathrm{T}-\mathrm{AOC}=$ total antioxidant capacity; TNF = tumor necrosis factor; IFN = interferon; SEM = standard error of means.

Table 3 Effect of alfalfa silage (AS) and mulberry leaf silage (MS) based diets on rumen fermentation characteristics in lambs 


\begin{tabular}{|lllll|}
\hline Items & \multicolumn{3}{l}{ Treatments } \\
\cline { 2 - 5 } & AS & MS & SEM & $P$-value \\
\hline $\mathrm{pH}$ & 5.88 & 5.64 & 0.231 & 0.42 \\
\hline Ammonia-N, mg/100mL & 24.1 & 31.3 & 5.46 & 0.40 \\
\hline Total VFA, mmol/L & 110.6 & 128.2 & 18.42 & 0.54 \\
\hline Molar proportion, \% & & & & \\
\hline Acetate & 64.8 & 62.0 & 2.42 & 0.44 \\
\hline Propionate & 19.5 & 24.3 & 2.87 & 0.29 \\
\hline Butyrate & 12.2 & 10.7 & 1.31 & 0.45 \\
\hline Valerate & 1.06 & 1.27 & 0.135 & 0.33 \\
\hline Isobutyrate & 0.85 & 0.49 & 0.125 & 0.09 \\
\hline Isovalerate & 1.51 & 1.29 & 0.330 & 0.66 \\
\hline Acetate:Propionate & 3.35 & 3.34 & 0.733 & 0.99 \\
\hline
\end{tabular}

VFA = volatile fatty acids; SEM = standard error of means.

\section{Figures}


A

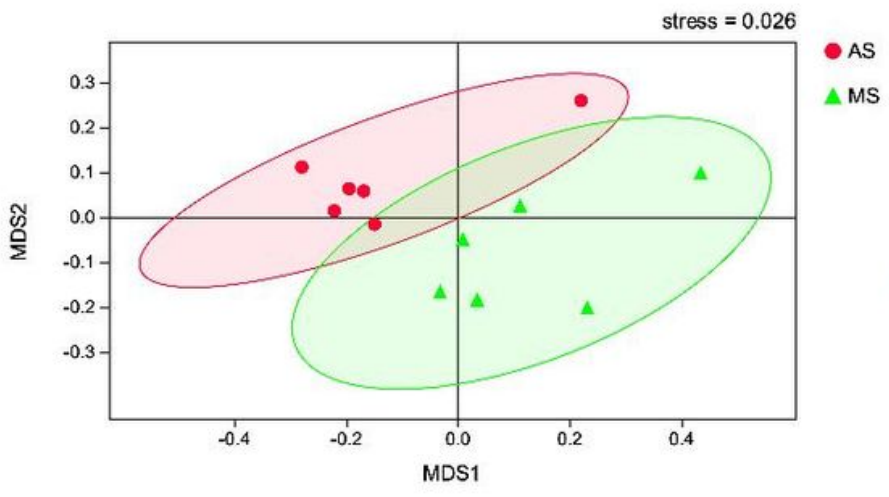

B

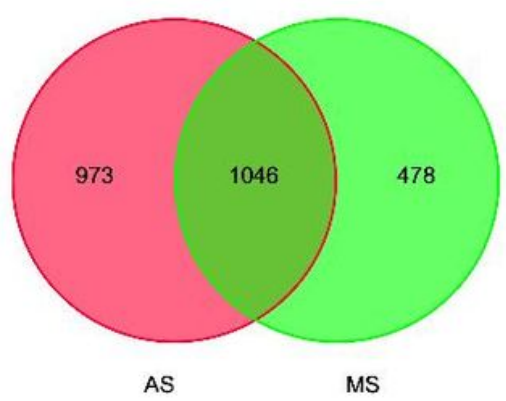

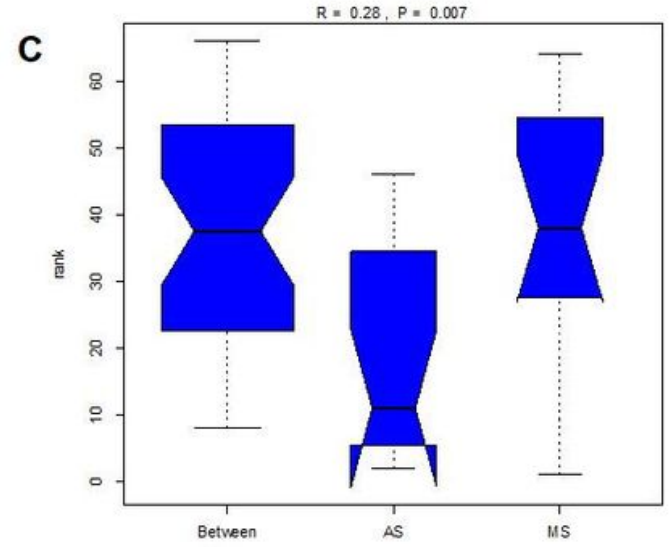

\section{Figure 1}

Unweighted non-metric multi-dimensional scaling (NMDS) of taxonomical classifications of bacterial communities (A). Venn diagram illustrating overlap of microbial operational taxonomic units (OTUs) between the two groups (B). Anosim (analysis of similarities) based on bray-curtis distances between the two groups (C). AS, alfalfa silage based diet; MS, mulberry leaf silage based diets. 
A

Phylum

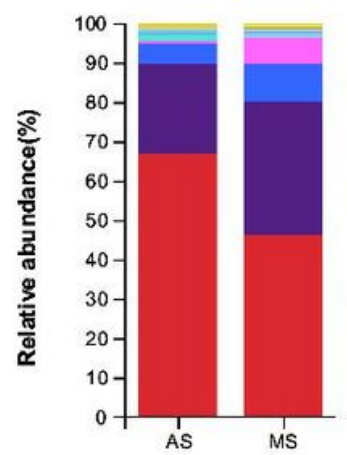

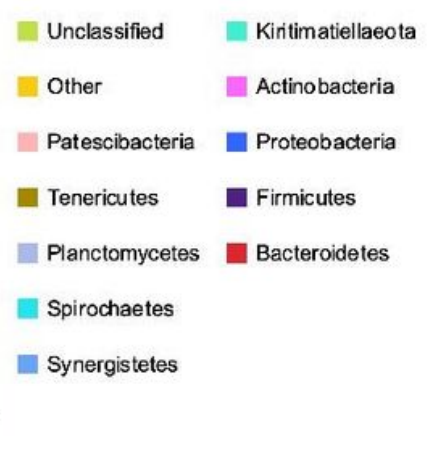

B

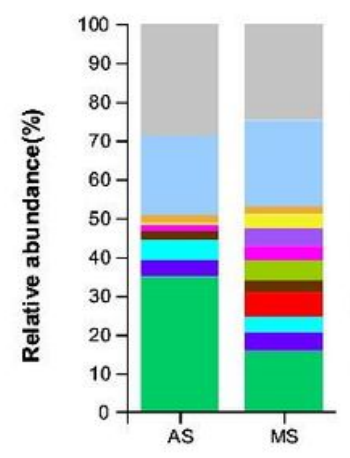

Unclassified

Other

In Veillonellaceae_UCG-001

[CRuminococus_2

Succinivibrionaceae_UCG-001

Prevotellaceae_UCG-001

Bifidobacterium
Succiniclasticum

[ Prevotella_7

Rikenellaceae_RC9_gut_group

- Selenomonas_1

[ Prevotella_1

\section{Figure 2}

Relative abundance of bacteria community proportions at phylum (A) and genus (B) level between alfalfa silage (AS) and mulberry leaf silage (MS) treatments (as a percentage of the total sequence).

A

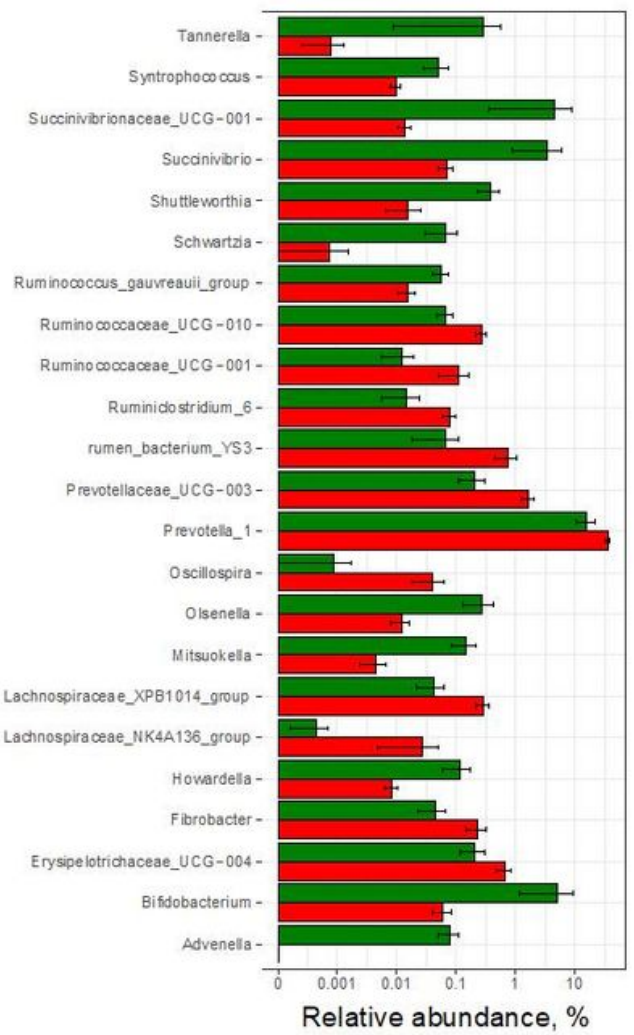

B
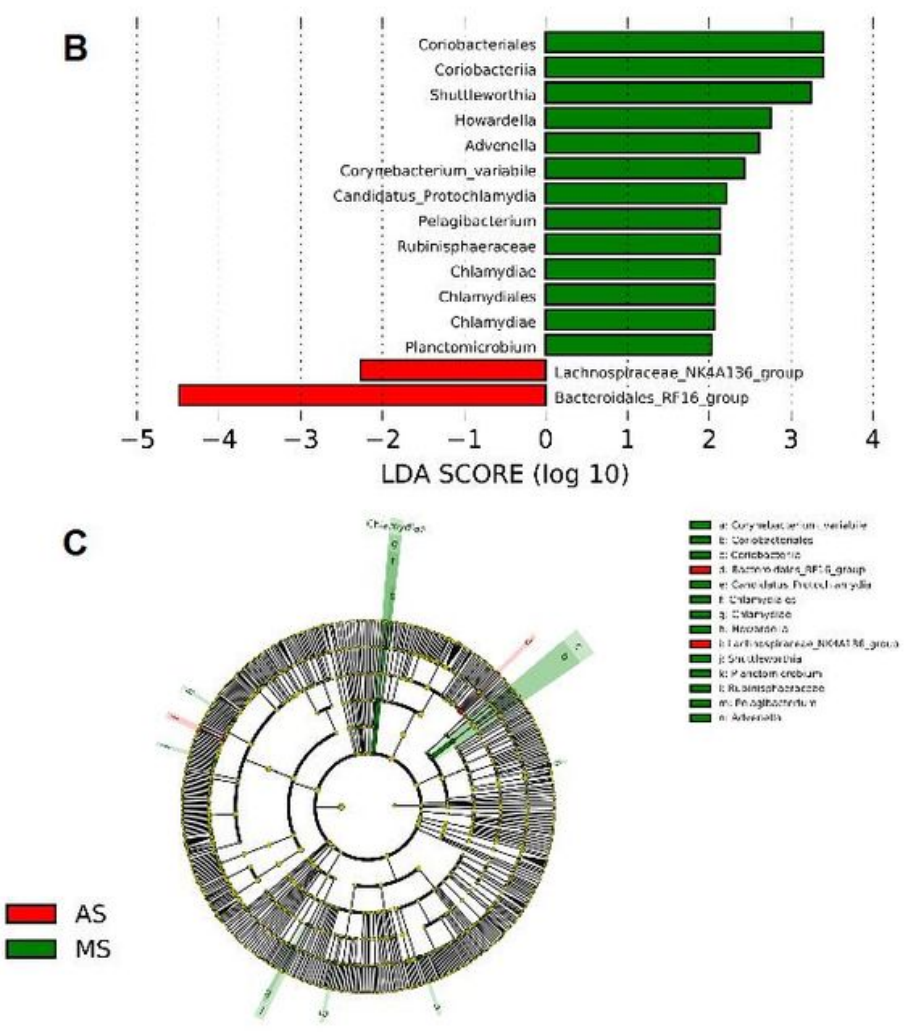


\section{Figure 3}

The core specific bacterial biomarker. Significantly different genus bacteria between alfalfa silage (AS) and mulberry leaf silage (MS) treatments (B) were tested by Wilcoxon rank-sum test with P-value of < 0.05 (A). LEfSe (Linear discriminant analysis Effect Size) determined using the Wilcoxon rank sum test ( $p$ $<0.05$ ) with a linear discriminant analysis (LDA) score analysis shows differentially abundant bacteria communities between alfalfa silage (AS) and mulberry leaf silage (MS) treatments (B); The cladogram shows the taxonomic levels represented by rings with six layers from the inside of this plot to the outside, corresponding to six levels of taxonomy (kingdom, phylum, class, order, family, and genus). Each node (small circle) represents a taxon (C). Green and red columns or nodes represent the bacteria with the significant higher relative abundance in MS and AS group, respectively.

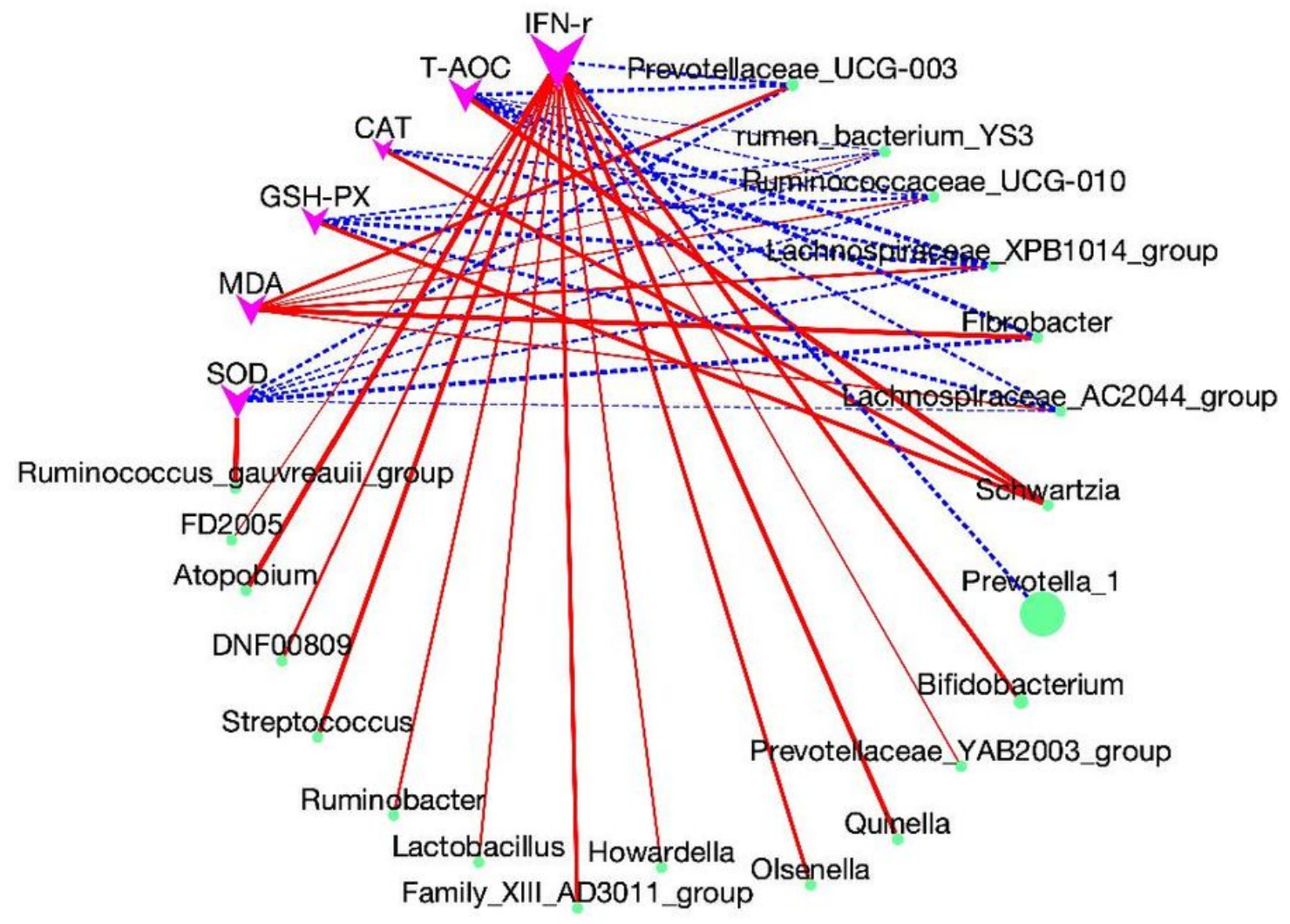

\section{Figure 4}

Interactions between rumen different microbiome and serum differential antioxidant and immune parameters. Pearson's correlations network showing relationships between rumen microbiota and mulberry leaf silage-associated index in serum. Only strong correlations $(r>0.59$ or $r<-0.59, P<0.05)$ were showed in the correlation networks. Red straight line, positive correlation ( $r>0.59)$; blue dotted line, negative correlation $(r<-0.59)$. The thicker the line, the higher the correlation. Microbes are shown by pink $\mathrm{V}$-shaped nodes, and serum parameters are shown by green round nodes.

\section{Supplementary Files}


This is a list of supplementary files associated with this preprint. Click to download.

- Coverletter.docx

- WangBSupplementaryMaterial.docx 